02

\title{
Н-комплексы 1,2-нафтохинона с молекулами воды в водном растворе и их влияние на сдвиги полос поглощения
}

\author{
(C) C.H. Цеплина, E.E. Цеплин ब \\ Институт фризики молекул и кристаллов УФИЦ РАН, \\ 450075 Уфа, Россия \\ ฯ e-mail: tzeplin@mail.ru \\ Поступила в редакцию 23.11.2020 г. \\ В окончательной редакции 02.02.2021 г. \\ Принята к публикации 05.02.2021 г.
}

Получены спектры оптического поглощения 1,2-нафтохинона в неполярном (н-гексан) и полярном (вода) растворителях. Показано, что применение квантово-химических расчетов на основе теории временной зависимости функционала плотности (TD-DFT B3LYP/6-311+G(d,p)) с моделью поляризуемого континуума (PCM) для расчета 1,2-нафтохинона в растворе $H$-гексана и расчета водородного комплекса 1,2-нафтохинона с двумя молекулами воды в водной среде хорошо описывает сдвиги полос поглощения 1,2-нафтохинона в растворе воды по сравнению с раствором в $t$-гексане. На основе анализа отклонений расчетных сдвигов полос от экспериментальных рассмотрен вопрос об образовании в водном растворе водородных комплексов 1,2-нафтохинона с $n$ молекулами воды $(n=1-4)$.

Ключевые слова: спектры поглощения, 1,2-нафтохинон, водородный комплекс, влияние растворителя.

DOI: $10.21883 /$ OS.2021.05.50884.40-20

\section{Введение}

Учет влияния растворителя является важной задачей для многих областей научной и технической деятельности, таких как синтетическая химия, молекулярная биология и материаловедение, поскольку большинство химических реакций, а также многие структурные исследования происходят в различных растворах [1-3]. Для описания взаимодействия молекул растворенного вещества с молекулами растворителя предложено несколько теоретических моделей $[4,5]$, одной из которых является модель поляризуемого континуума (РСМ) [6,7]. Применение этой модели с теорией временной зависимости функционала плотности (TD-DFT) хорошо описывает сдвиги полос поглощения и люминесценции в различных растворителях [8-13]. При этом в полярных растворителях наряду с учетом общего влияния растворителя необходимо учитывать, что часть молекул растворителя образуют водородные связи с молекулой растворенного вещества [4,12-17]. Возникает вопрос определения количества таких молекул для лучшего воспроизведения экспериментальных данных, в частности сдвигов полос поглощения. Для образования водородных связей необходимо наличие во взаимодействующих молекулах донорно-акцепторных функциональных групп [1]. Например, для ряда соединений с карбонильными группами и гидроксильной группой [12-13,15-17] было показано, что хорошее воспроизведение экспериментальных сдвигов полос поглощения в растворе метанола по сравнению с неполярным растворителем (н-гексаном), наблюдается при расчете водородного комплекса моле- кулы с таким количеством молекул метанола, которое равно количеству функциональных групп в молекуле.

Наибольший практический интерес представляют водные растворы, в связи с чем сольватная оболочка, образуемая молекулами воды, является наиболее исследуемой $[4,9,14,18-25]$. Вместе с тем именно определение числа молекул воды, образующих водородный комплекс с молекулой растворенного вещества, вызывает наибольше расхождение в научной литературе. Так, в одной из первых работ [19] по исследованию первичной водной сольватной оболочки авторы на основе теоретической оценки энергии системы установили, что для молекулы тимина, содержащей четыре функциональные группы (две карбонильные и две $\mathrm{N}-\mathrm{H}$ ), более устойчивый гидрат образуется из трех, а не четырех молекул воды. В то же время в работе [14] для воспроизведения влияния растворителя на полосы поглощения урацила, содержащего такие же функциональные группы, на основе данных ЯМР были выбраны четыре молекулы воды. Для той же молекулы урацила в работе [21] с помощью молекулярной динамики (метод „Car-Parrinello“) было получено, что первичная сольватная оболочка урацила состоит из девяти молекул воды, шесть из которых связаны с молекулой урацила водородными связями. На основе теоретической оценки $a b$ initio (метод MP2) энергии связи молекулы урацила в комплексе с двумя, тремя и четырьмя молекулами воды в работе [22] было показано, что наиболее устойчивый комплекс образуется с двумя молекулами воды. Аналогичное расхождение при определении числа молекул воды, образующих водородные комплексы, наблюдается и для других соединений, например цитозина [23-25]. 
Хорошо известно, что сдвиги полос поглощения при переходе от неполярных растворителей к полярным обусловлены изменением межмолекулярного взаимодействия между молекулами растворенного вещества и молекулами растворителя. При этом более сильное влияние на сдвиги полос оказывает образование молекулярных комплексов, в частности комплекса с образованием водородных связей [1]. Метод PCM TD-DFT хорошо описывает сдвиги полос поглощения и люминесценции в различных растворителях [8-13], обусловленные общими эффектами растворителя. Тогда для полярных растворителей, в которых образуются Н-комплексы, можно рассмотреть обратную задачу - определить число молекул растворителя, образующих Н-комплекс, и его структуру на основе теоретического воспроизведения экспериментальных сдвигов полос поглощения в пределах погрешности данной теории.

Карбонильная группа является структурным элементом большинства биологически активных соединений (элементы ДНК, РНК, белки и др.), в том числе и группы хинонов (убихинон, пластохинон, витамин К1), в связи с чем вопрос образования Н-комплексов соединений, содержащих карбонильные группы, с молекулами воды остается наиболее актуальным.

В настоящей работе рассматриваются сдвиги полос поглощения 1,2-нафтохинона (12NQ) в водном растворе (полярный растворитель) по сравнению с раствором в $\boldsymbol{H}$-гексане (неполярный растворитель). При моделировании общих эффектов растворителя использовалась модель РСМ, а для учета специфических взаимодействий (водородных связей) рассмотрено образование Н-комплекса $12 \mathrm{NQ}$ с различным числом молекул воды $(n=1-4)$. Выбор 12NQ в качестве объекта исследования обусловлен тем, что по спектрам поглощения этого хинона можно экспериментально определять сдвиги сразу для четырех полос поглощения - гипсохромного сдвига первой полосы $n \pi^{*}$ и батохромного для следующих трех полос $\pi \pi^{*}[17]$.

Спектры оптического поглощения 12NQ были получены ранее другими авторами [26-30] и авторами настоящей работы в растворах $\boldsymbol{H}$-гексана и метанола [17]. В работе [17] также выполнена интерпретация спектра поглощения $12 \mathrm{NQ}$.

\section{Экспериментальная часть}

Спектры поглощения 1,2-нафтохинона (Aldrich, $\geq 97 \%$ ) записаны в водном растворе и в растворе н-гексана (Panreac, $\geq 95 \%$ ) в стандартной кварцевой кювете $10 \mathrm{~mm}$ на спектрофотометре Shimadzu UV-2401 со скоростью сканирования $210 \mathrm{~nm} / \mathrm{min}$ и спектральной шириной щели $1 \mathrm{~nm}$. Концентрация раствора 1,2-нафтохинона в $\boldsymbol{H}$-гексане $0.63 \cdot 10^{-4} \mathrm{~mol} / 1$, в воде $-0.55 \cdot 10^{-4} \mathrm{~mol} / \mathrm{l}$, при записи первой слабо интенсивной полосы в $H$-гексане $-1.3 \cdot 10^{-4} \mathrm{~mol} / \mathrm{l}$. Соединение 1,2-нафтохинон плохо растворяется в
$\boldsymbol{H}$-гексане, поэтому в настоящей работе растворение проводилось с помощью магнитной мешалки в течение двух часов при температуре $315 \mathrm{~K}$. Значения молярных коэффициентов поглощения для полос поглощения 1,2-нафтохинона в $H$-гексане были скорректированы по сравнению с работой [17].

Квантово-химические расчеты выполняли методом электронной плотности (DFT) с обменно-корреляционным функционалом B3LYP [31-33] в базисе 6-311+G(d,p) с использованием программы GAUSSIAN 09 [34]. Учет влияния растворителей $\boldsymbol{H}$-гексана и воды осуществляли на основе модели РСМ. Локализацию стационарных точек на поверхности потенциальной энергии (ППЭ) осуществляли с полной оптимизацией всех геометрических параметров. При отсутствии мнимых частот стационарные точки ППЭ относили к минимумам, а при наличии одной мнимой частоты - к переходному состоянию. Расчеты электронных спектров были выполнены методом TD-DFT на основе функционала B3LYP и того же базисного набора 6-311+G(d,p) для 60 возбужденных синглетных состояний. Для визуализации геометрии и симуляции расчетных электронных спектров применялась программа Chemcraft 1.7 [35]. Расчетный электронный спектр симулировался с помощью кривых Лоренца с шириной на полувысоте $0.3 \mathrm{eV}$.

\section{Результаты и обсуждение}

Спектры оптического поглощения 12NQ, записанные в растворе $\boldsymbol{H}$-гексана и в водном растворе, представлены на рис. 1, $a$. Расчетные электронные спектры молекулы $12 \mathrm{NQ}$ в $н$-гексане и ее водородного комплекса с двумя молекулами воды в водном растворе показаны на рис. $1, b$. Отнесение спектров поглощения $12 \mathrm{NQ}$ в растворах $н$-гексана [17] и воды, а также экспериментальные и расчетные величины сдвига полос приведены в табл. 1.

Как видно из рис. 1 и табл. 1, расчет электронных спектров молекулы $12 \mathrm{NQ}$ в $H$-гексане и ее водородного комплекса с двумя молекулами воды в воде хорошо воспроизводит как сами экспериментальные спектры поглощения $12 \mathrm{NQ}$ в этих растворителях, так и наблюдаемые экспериментальные сдвиги полос. Из рис. 1, $a$ видно, что в полярном растворителе воде первая полоса поглощения, соответствующая $n_{\mathrm{O}}^{-} \rightarrow \pi_{(\mathrm{C}=\mathrm{O}) 3}^{*}$-переходу, испытывает гипсохромный сдвиг, а для следующих трех полос поглощения, соответствующих преимущественно $\pi \pi^{*}$-переходам, наблюдается батохромный сдвиг. В результате разного направления этих сдвигов первая полоса поглощения 12NQ в водном растворе оказывается частично перекрытой более интенсивной второй полосой. Поэтому максимум первой полосы поглощения 12NQ в воде определен приблизительно по точке перегиба кривой поглощения, оценка сдвига этой полосы проведена для адиабатического перехода (рис. 1, $a$, табл. 1).

При изучении комплексов молекулы $12 \mathrm{NQ}$ с $n$ молекулами воды $(n=1-4)$ были рассчитаны четыре ком- 
Таблица 1. Отнесение спектров поглощения 1,2-нафтохинона в растворе $н$-гексана и водном растворе на основании расчета РСМ TD-DFT B3LYP/6-311+G(d,p) 1,2-нафтохинона в $t$-гексане и водородного комплекса 1,2-нафтохинона с двумя молекулами воды в водном растворе

\begin{tabular}{|c|c|c|c|c|c|c|c|c|c|c|c|}
\hline \multirow{2}{*}{$S$} & \multirow{2}{*}{$3 \mathrm{MO}-\mathrm{BMO}$} & \multicolumn{2}{|c|}{$\mu$-гексан } & \multicolumn{2}{|c|}{ Вода } & \multirow{2}{*}{$\Delta E^{\exp }$} & \multicolumn{2}{|c|}{ РСМ $н$-гексан } & \multicolumn{2}{|c|}{ РСМ вода } & \multirow{2}{*}{$\Delta E^{\text {calc }}$} \\
\hline & & $E^{\exp }$ & $\varepsilon$ & $E^{\exp }$ & $\varepsilon$ & & $E^{\text {calc }}$ & $f$ & $E^{\text {calc }}$ & $f$ & \\
\hline$S_{1}$ & $n_{\mathrm{O}}^{-} \rightarrow \pi_{(\mathrm{C}=\mathrm{O}) 3}^{*}$ & $\begin{array}{c}2.29 \\
\left(2.13^{\mathrm{a}}\right)\end{array}$ & 0.01 & $\begin{array}{r}\sim 2.5 \\
\left(2.29^{\mathrm{a}}\right)\end{array}$ & 0.04 & $0.16^{\mathrm{a}}$ & 2.13 & 0 & 2.33 & 0 & 0.20 \\
\hline $\begin{array}{l}S_{2} \\
S_{3}\end{array}$ & $\begin{array}{l}\pi_{3} \rightarrow \pi_{(\mathrm{C}=\mathrm{O}) 3}^{*} \\
n_{\mathrm{O}}^{+} \rightarrow \pi_{\mathrm{C}=0) 3}^{*}\end{array}$ & 3.24 & 0.24 & 3.01 & 0.24 & -0.23 & $\begin{array}{l}2.92 \\
3.42\end{array}$ & $\begin{array}{l}0.0654 \\
0.0002\end{array}$ & $\begin{array}{l}2.64 \\
3.59\end{array}$ & $\begin{array}{c}0.0513 \\
0\end{array}$ & -0.28 \\
\hline$S_{4}$ & $\begin{aligned} \mathrm{O}_{\mathrm{O}} & \rightarrow \pi_{(\mathrm{C}=0) 3}^{*} \\
\pi_{2} & \rightarrow \pi_{(\mathrm{C}=0) 3}\end{aligned}$ & 3.79 & 0.19 & 3.56 & 0.31 & -0.23 & 3.65 & 0.0874 & 3.41 & 0.1121 & -0.24 \\
\hline$S_{5}$ & $n_{\mathrm{O}}^{-} \rightarrow \pi_{4}^{*}$ & & & & & & 4.47 & 0.0001 & 4.87 & 0.0001 & \\
\hline$S_{6}$ & $\pi_{(\mathrm{C}=\mathrm{C})} \rightarrow \pi_{(\mathrm{C}=\mathrm{O}) 3}^{*}$ & & & $\sim 4.5$ & $\sim 0.2$ & & 4.91 & 0.0460 & 4.70 & 0.0293 & \\
\hline$S_{7}$ & $n_{\mathrm{O}}^{-} \rightarrow \pi_{5}^{*}$ & & & & & & 5.29 & 0 & 5.75 & 0.0008 & \\
\hline$S_{8}$ & $\pi_{3} \rightarrow \pi_{4}^{*}$ & $\begin{array}{c}4.94 \\
\left(5.05^{\mathrm{b}}\right)\end{array}$ & 2.61 & $\begin{array}{c}4.84 \\
\left(4.94^{\mathrm{b}}\right)\end{array}$ & 2.53 & $\begin{array}{c}-0.10 \\
\left(-0.11^{\mathrm{b}}\right)\end{array}$ & 5.13 & 0.5939 & 5.11 & 0.6311 & -0.02 \\
\hline
\end{tabular}

Примечание. $S$ - номера возбужденных синглетных состояний (порядок состояний указан для газовой фазы [17]); 3МО-ВМО - тип МО, вклад перехода между которыми в данное состояние наибольший; $E^{\text {calc }}$ и $E^{\text {exp }}-$ расчетные и экспериментальные вертикальные энергии переходов (eV); $f$ - сила осциллятора; $\varepsilon-$ молярный показатель поглощения $\left(10^{3} \mathrm{~m}^{2} / \mathrm{mol}\right) ; \Delta E^{\text {exp }}, \Delta E^{\text {calc }}-$ энергии экспериментальных и расчетных сдвигов полос поглощения 1,2-нафтохинона в воде по сравнению с $\boldsymbol{t}$-гексаном $(\mathrm{eV})$.

а Энергия указана для адиабатического перехода.

b Оценка сдвига сделана по второму колебательному пику полосы.
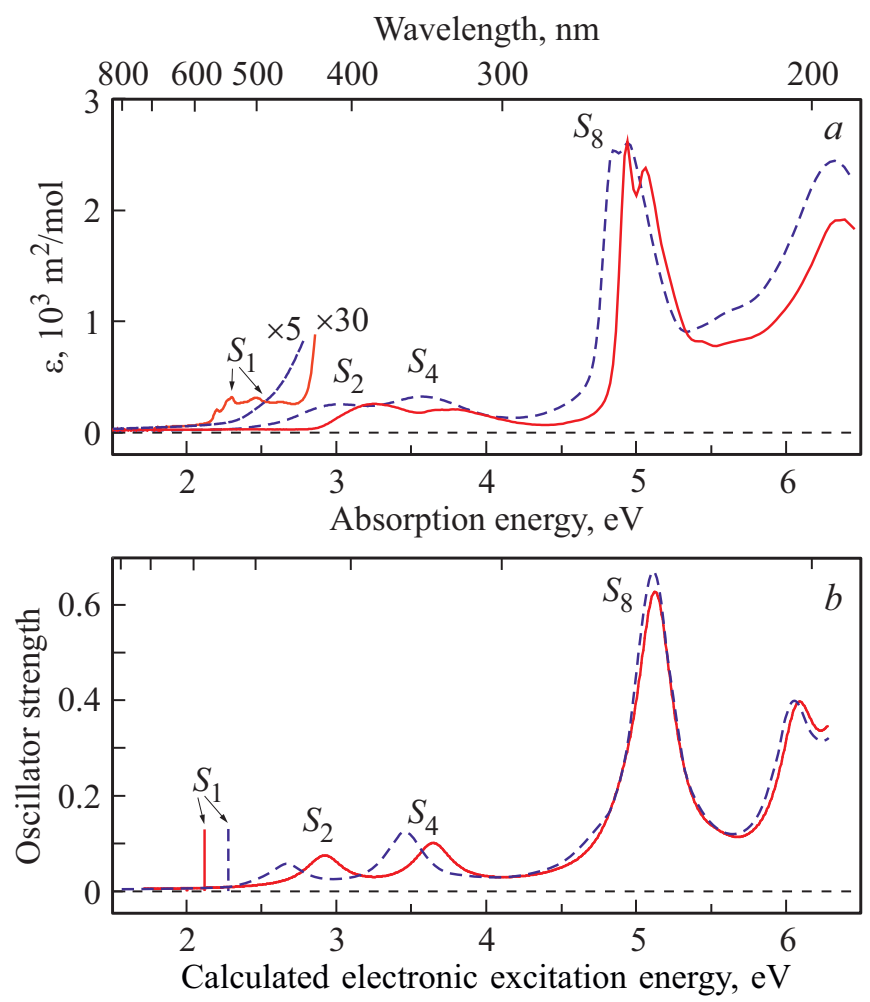

Рис. 1. (a) Спектры оптического поглощения 1,2-нафтохинона в растворах $\boldsymbol{H}$-гексана (сплошная кривая) и воды (штриховая кривая). (b) Расчет методом PCM TD-DFT $\mathrm{B} 3 \mathrm{LYP} / 6-311+\mathrm{G}(\mathrm{d}, \mathrm{p})$ электронных спектров 1,2-нафтохинона в $\boldsymbol{H}$-гексане (сплошная кривая) и водородного комплекса 1,2-нафтохинона с двумя молекулами воды в воде (штриховая кривая). плекса для каждого $n$ (рис. 2-5). При дальнейшем рассмотрении из них были взяты те, которые имеют минимальную полную энергию. Такие Н-комплексы далее называются основными. Их структура представлена на рис. $2, a-5, a$. Как видно из рис. $3, a-5, a$, наиболее стабильным комплексом $12 \mathrm{NQ}$ с $n$ молекулами воды $(n=2-4)$ является такой, когда молекулы воды образуют замкнутую структуру, связывающую цепочкой водородных связей атомы кислорода молекулы 12NQ. Затем для этих основных Н-комплексов (рис. 2, $a-5, a$ ) были рассчитаны энергии электронных переходов, вычислены сдвиги электронных переходов в воде по сравнению с $H$-гексаном $\left(\Delta E^{\text {calc }}\right)$ и сопоставлены с экспериментальными сдвигами полос $\left(\Delta E^{\exp }\right)$. В табл. 2 для четырех полос поглощения молекулы $12 \mathrm{NQ}$ (соответствующих переходам в состояния $S_{1}, S_{2}, S_{4}$ и $\left.S_{8}\right)$ приведены значения $\Delta E^{\exp }, \Delta E^{\text {calc }}$ и значения отклонений расчетных сдвигов электронных переходов от экспериментальных $\left(\Delta(\Delta E)=\Delta E^{\exp }-\Delta E^{\text {calc }}\right)$ для комплексов с различным числом молекул воды $(n=1-4)$. Как видно из табл. 2 , наименьшие отклонения расчетных сдвигов от экспериментальных для первых двух полос $S_{1}$ и $S_{2}$ наблюдаются при образовании водородного комплекса $12 \mathrm{NQ}$ с одной молекулой воды, а для двух других полос $S_{4}$ и $S_{8}-$ с двумя молекулами воды и более. Однако образование водородного комплекса $12 \mathrm{NQ}$ только с одной молекулой воды маловероятно, поскольку в комплексе, содержащем более одной молекулы воды, согласно расчетным данным образуются минимум две водородные связи между двумя группами $\mathrm{C}=\mathrm{O}$ и молекулами воды (рис. 2-5). Поэтому наилучшее воспроизведение экспериментальных сдвигов всех рассматриваемых полос дает расчет Н-комплекса 12NQ с двумя (рис. 3,a) и четырьмя 

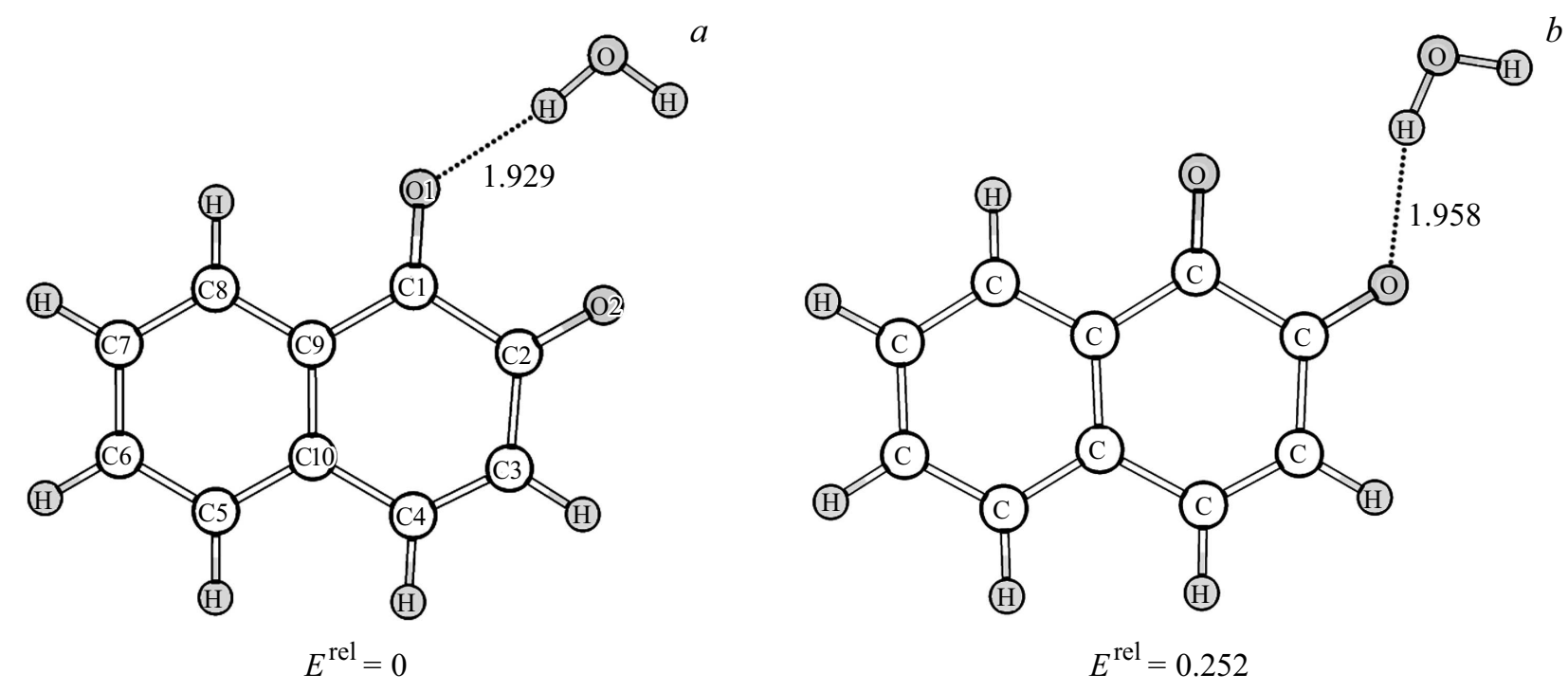

$b$

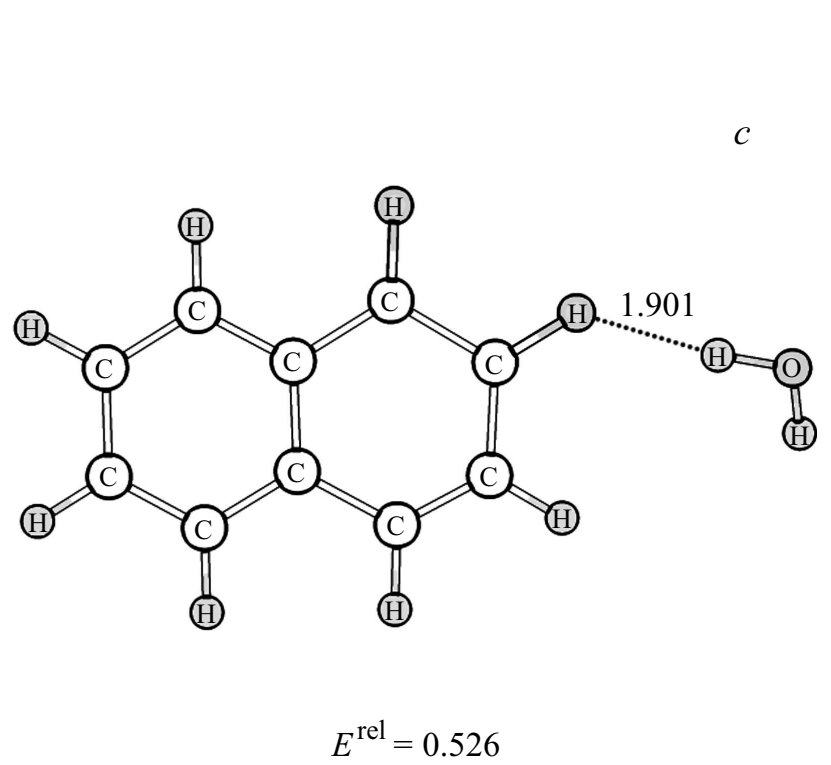<smiles>O=C1OC2(OC1=O)C(=O)OC(=O)C2=O</smiles>

Рис. 2. Оптимизированные геометрические структуры водородного комплекса 1,2-нафтохинона с одной молекулой воды. На рис. 2, $а$ приведено обозначение атомов молекулы 1,2-нафтохинона. Рядом с пунктирными линями, обозначающими водородные связи между атомами молекулы $12 \mathrm{NQ}$ и молекулами воды, поставлены числа, обозначающие длину водородной связи в ангстремах. $E^{\text {rel }}$ - энергия Н-комплекса относительно энергии основного Н-комплекса $(\mathrm{kcal} / \mathrm{mol})$.

(рис. 5, a) молекулами воды. Это свидетельствует о том, что структуры этих комплексов наиболее близки тем структурам, которые образуются молекулой 12NQ и частью сольватной оболочки в водном растворе. В настоящей работе при отнесении спектра поглощения 12NQ в воде использовались данные расчета водородного комплекса $12 \mathrm{NQ}$ с двумя молекулами воды (табл. 1, рис. 3,a). Характеристики водородных связей в основных Н-комплексах 1,2-нафтохинона с $n$ молекулами воды по данным расчета приведены в табл. 3 .

Из табл. 2 видно, что теоретический учет влияния континуума воды предсказывает для первых трех полос поглощения 12NQ значения сдвигов несколько меньшие, но близкие к экспериментальным: для $S_{1}-$ на $75 \%$, для
$S_{2}-$ на $78 \%$, для $S_{4}-$ на $65 \%$. Добавление к молекуле 12NQ одной и двух молекул воды, которые образуют с ней межмолекулярные водородные связи, увеличивает величину сдвига полос. При этом для комплекса с двумя молекулами воды предсказанные значения сдвигов оказываются завышенными по сравнению с экспериментальными: для $S_{1}-$ на $25 \%$, для $S_{2}-$ на $22 \%$, для $S_{4}-$ на $4 \%$. Исходя из этих данных можно сделать вывод, что водный континуум (общий эффект растворителя) предсказывает вклад в сдвиги полос поглощения молекулы $12 \mathrm{NQ}$, сопоставимый или даже более значимый в сравнении с вкладом от образования водородного комплекса (специфический эффект растворителя). Этот результат отличается от растворов хинонов в другом полярном 

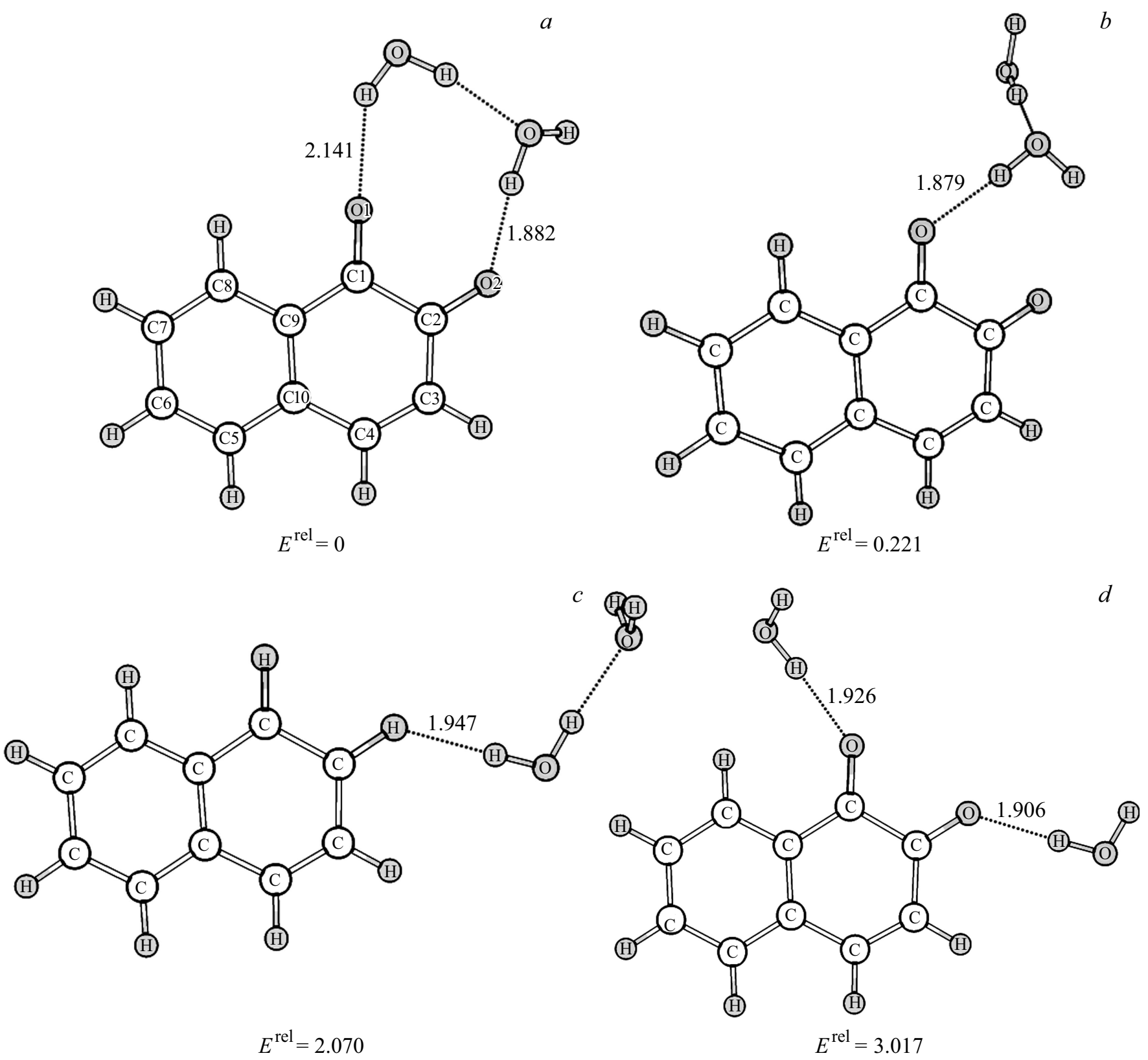

Рис. 3. Оптимизированные геометрические структуры водородного комплекса 1,2-нафтохинона с двумя молекулами воды. Рядом с пунктирными линями, обозначающими водородные связи между атомами 12NQ и молекулами воды, поставлены числа, обозначающие длину водородной связи в ангстремах. $E^{\text {rel }}-$ энергия Н-комплекса относительно энергии основного Н-комплекса с минимальной полной энергией $(\mathrm{kcal} / \mathrm{mol})$.

Таблица 2. Сдвиги полос поглощения 1,2-нафтохинона в водном растворе по сравнению с раствором в $\boldsymbol{H}$-гексане $(\mathrm{eV})$

\begin{tabular}{|c|c|c|c|c|c|c|c|c|c|c|c|}
\hline \multirow[t]{2}{*}{$S$} & \multirow[t]{2}{*}{$\Delta E^{\exp }$} & \multicolumn{2}{|c|}{$12 \mathrm{NQ}$} & \multicolumn{2}{|c|}{$12 \mathrm{NQ}+1 \mathrm{H}_{2} \mathrm{O}$} & \multicolumn{2}{|c|}{$12 \mathrm{NQ}+2 \mathrm{H}_{2} \mathrm{O}$} & \multicolumn{2}{|c|}{$12 \mathrm{NQ}+3 \mathrm{H}_{2} \mathrm{O}$} & \multicolumn{2}{|c|}{$12 \mathrm{NQ}+4 \mathrm{H}_{2} \mathrm{O}$} \\
\hline & & $\Delta E^{\text {calc }}$ & $\Delta(\Delta E)$ & $\Delta E^{\text {calc }}$ & $\Delta(\Delta E)$ & $\Delta E^{\text {calc }}$ & $\Delta(\Delta E)$ & $\Delta E^{\text {calc }}$ & $\Delta(\Delta E)$ & $\Delta E^{\mathrm{calc}}$ & $\Delta(\Delta E)$ \\
\hline$S_{1}$ & 0.16 & 0.12 & 0.04 & 0.17 & -0.01 & 0.20 & -0.04 & 0.24 & -0.08 & 0.20 & -0.04 \\
\hline$S_{2}$ & -0.23 & -0.18 & -0.05 & -0.25 & 0.02 & -0.28 & 0.05 & -0.29 & 0.06 & -0.28 & 0.05 \\
\hline$S_{4}$ & -0.23 & -0.15 & -0.08 & -0.19 & -0.04 & -0.24 & 0.01 & -0.24 & 0.01 & -0.24 & 0.01 \\
\hline$S_{8}$ & -0.10 & -0.01 & -0.09 & -0.01 & -0.09 & -0.02 & -0.08 & -0.02 & -0.08 & -0.02 & -0.08 \\
\hline
\end{tabular}

Примечание. $\Delta E^{\text {exp }}$ - экспериментальные значения сдвига полос; $\Delta E^{\text {calc }}\left(12 \mathrm{NQ}+n \mathrm{H}_{2} \mathrm{O}\right)-$ расчетные величины сдвига полос на основании расчета PCM TD-DFT B3LYP/6-311+G(d,p) для молекулы 1,2-нафтохинона и для основных водородных комплексов с $n=1-4$ молекулами воды; $\Delta(\Delta E)=\Delta E^{\exp }-\Delta E^{\text {calc }}$. 


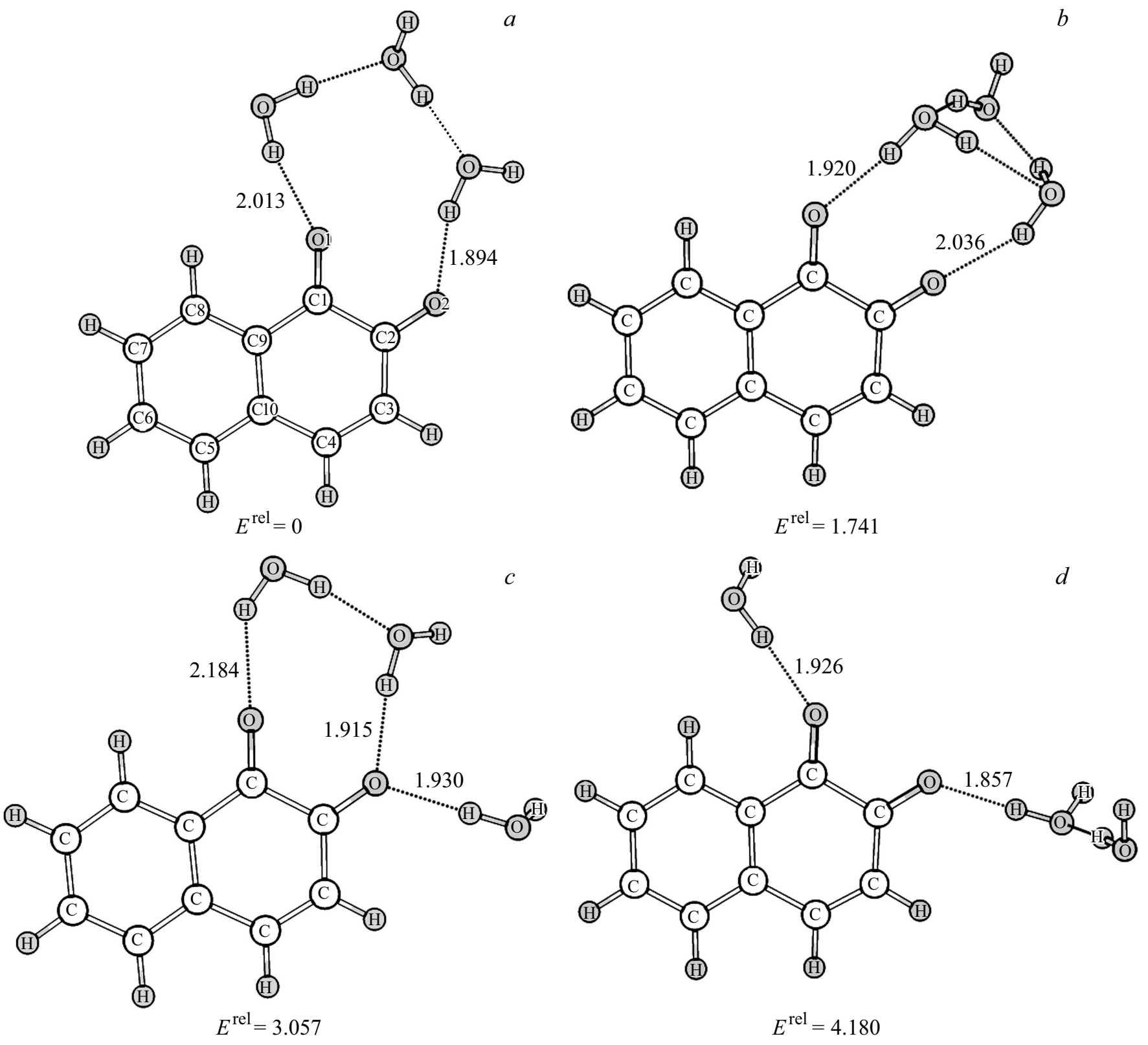

Рис. 4. Оптимизированные геометрические структуры водородного комплекса 1,2-нафтохинона с тремя молекулами воды. Рядом с пунктирными линями, обозначающими водородные связи между атомами 12NQ и молекулами воды, поставлены числа, обозначающие длину водородной связи в ангстремах. $E^{\text {rel }}-$ энергия Н-комплекса относительно энергии основного Н-комплекса с минимальной полной энергией $(\mathrm{kcal} / \mathrm{mol})$.

протонном растворителе - метаноле, в котором специфический эффект дает доминирующий вклад в сдвиги полос поглощения по сравнению с неполярным растворителем (н-гексаном) [14-16]. Что касается четвертой полосы $\left(S_{8}\right)$, которая в эксперименте подвергается батохромному сдвигу на $0.10 \mathrm{eV}$, как расчет $12 \mathrm{NQ}$ в воде, так и расчеты рассматриваемых в работе комплексов качественно воспроизводят сдвиг этой полосы, но количественное воспроизведение ее сдвига хуже $(0.02 \mathrm{eV})$, чем для других полос. Первые три рассматриваемых полосы поглощения соответствуют электронным переходам с участием орбиталей $n_{\mathrm{O}}^{-}$и $\pi_{(\mathrm{C}=\mathrm{O}) 3}^{*}$, имеющих основную часть электронной плотности на атомах карбонильной группы. В отличие от них четвертая полоса обусловлена электронным переходом между орбиталями $\pi_{3} \rightarrow \pi_{4}^{*}$, электронная плотность на которых локализована преимущественно на атомах углерода молекулы, и ее вклад на атомах карбонильных групп значительно меньше. Поэтому образование водородных комплексов между атомами кислорода молекулы 12NQ и молекулами воды будет слабо влиять на энергию этого перехода, как видно из данных табл. 2. Основной вклад в сдвиг этой полосы должен быть обусловлен общим эффектом растворителя. Но поскольку расчет $12 \mathrm{NQ}$ в воде также дает неудо- 


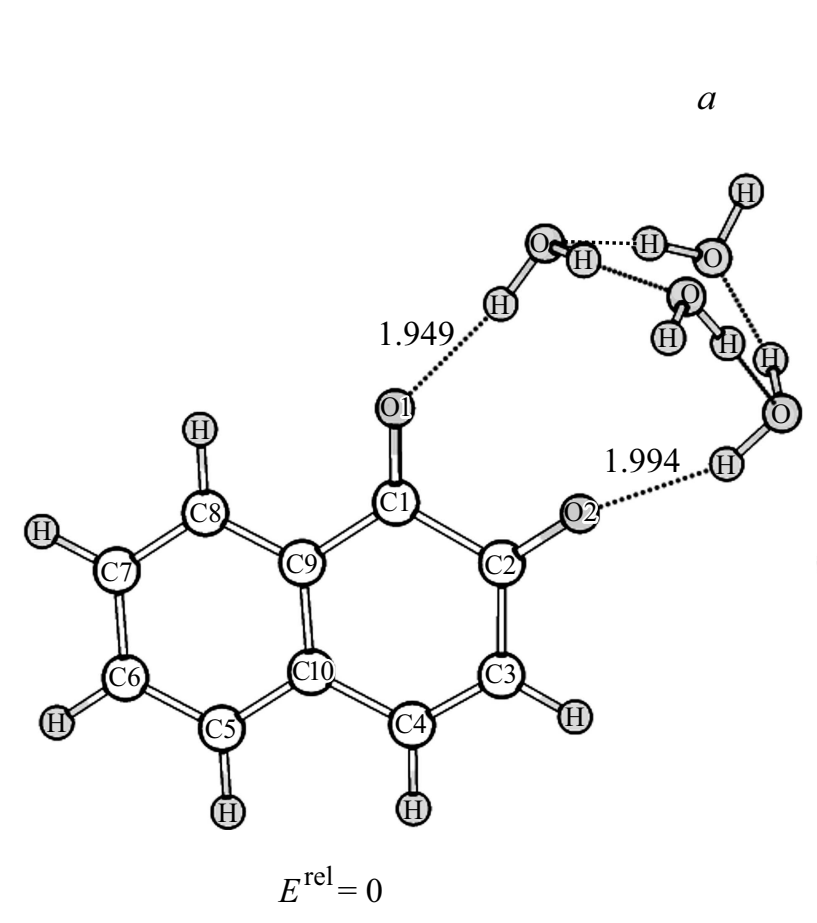

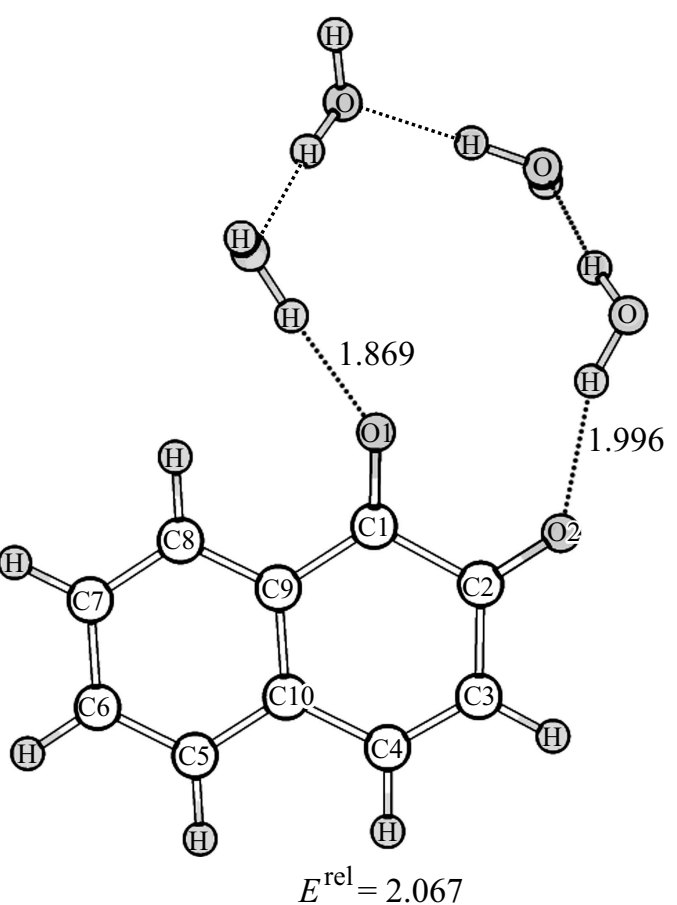

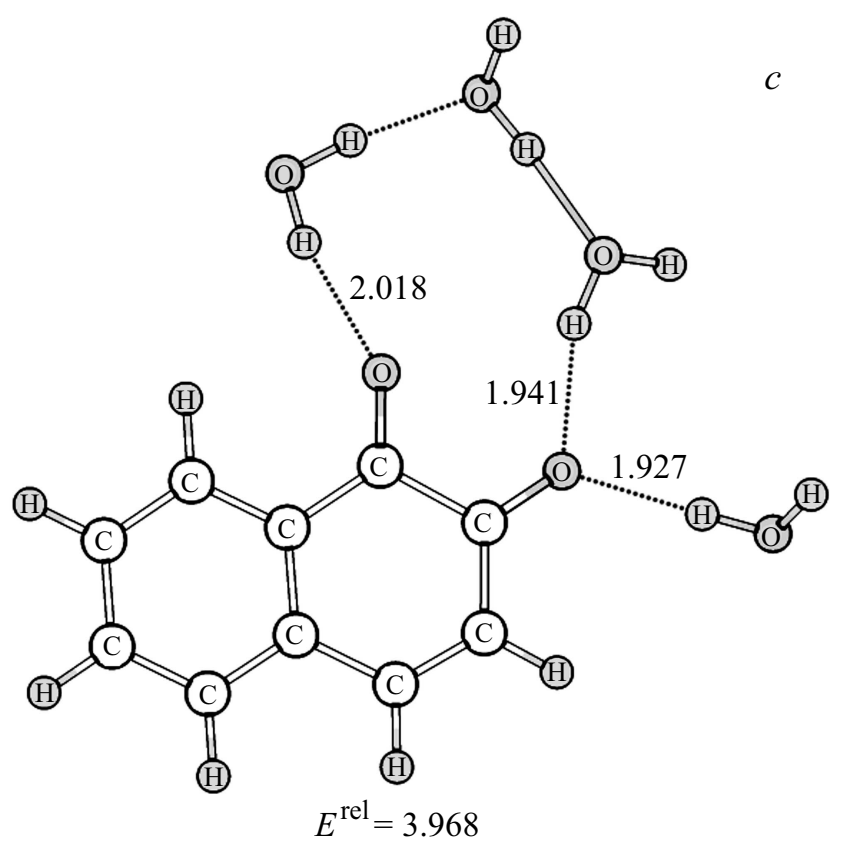

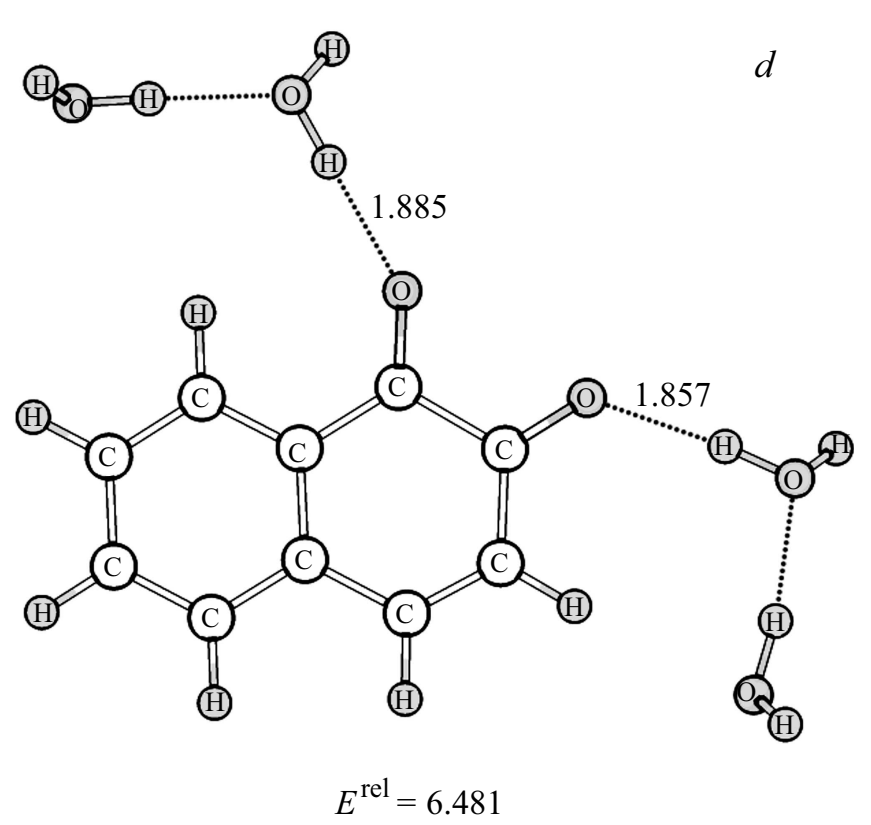

Рис. 5. Оптимизированные геометрические структуры водородного комплекса 1,2-нафтохинона с четырьмя молекулами воды. Рядом с пунктирными линями, обозначающими водородные связи между атомами $12 \mathrm{NQ}$ и молекулами воды, поставлены числа, обозначающие длину водородной связи в ангстремах. $E^{\text {rel }}$ - энергия Н-комплекса относительно энергии основного Н-комплекса с минимальной полной энергией $(\mathrm{kcal} / \mathrm{mol})$.

влетворительное воспроизведение экспериментального сдвига, можно предположить, что наблюдаемое плохое воспроизведение обусловлено погрешностью модели РСМ, в которой результаты моделирования изменения электронной плотности на внутренних атомах углерода при смене растворителя будут хуже, чем для внешних атомов кислорода. В целом данный вопрос остается открытым и требует дальнейших исследований.

\section{Выводы}

Показано, что квантово-химический расчет электронного спектра молекулы $12 \mathrm{NQ}$ методом TD-DFT B3LYP/6-311+G(d,p) с применением модели РСМ хорошо воспроизводит спектр поглощения $12 \mathrm{NQ}$ в растворе $H$-гексана, а расчет этим методом водородного комплекса $12 \mathrm{NQ}$ с двумя молекулами воды - в водном растворе. 
Таблица 3. Характеристики водородных связей в основных Н-комплексах 1,2-нафтохинона с $n$ молекулами воды $(n=1-4)$ в водном растворе по данным расчета PCM TD-DFT B3LYP/6-311+G(d,p)

\begin{tabular}{c|c|c|c|c}
\hline Параметр & $12 \mathrm{NQ}+1 \mathrm{H}_{2} \mathrm{O}$ & $12 \mathrm{NQ}+2 \mathrm{H}_{2} \mathrm{O}$ & $12 \mathrm{NQ}+3 \mathrm{H}_{2} \mathrm{O}$ & $12 \mathrm{NQ}^{2} 4 \mathrm{H}_{2} \mathrm{O}$ \\
\hline$R(\mathrm{O} 1 \ldots \mathrm{O})$ & 2.896 & 2.997 & 2.957 & 2.913 \\
$R(\mathrm{O} 2 \ldots \mathrm{O})$ & - & 2.854 & 2.846 & 2.908 \\
$\angle(=\mathrm{O} 1 \ldots \mathrm{H}-\mathrm{O})$ & 172.0 & 146.9 & 164.4 & 170.4 \\
$\angle(=\mathrm{O} 2 \ldots \mathrm{H}-\mathrm{O})$ & - & 173.7 & 164.3 & 156.3 \\
$\Delta \mathrm{H}(=\mathrm{O} 1 \ldots \mathrm{H}-\mathrm{O})$ & 2.46 & $3.49^{\mathrm{a}}$ & $4.91^{\mathrm{b}}$ & $4.27^{\mathrm{c}}$ \\
$\Delta \mathrm{H}(=\mathrm{O} 2 \ldots \mathrm{H}-\mathrm{O})$ & - & &
\end{tabular}

Примечание. Нумерация атомов и структура комплексов приведены на рис. $2, a-5, a . R(\mathrm{O} 1$. . O $)-$ расстояние $(\AA)$ между атомом кислорода О1 молекулы $12 \mathrm{NQ}$ и атомом кислорода молекулы воды, образующей водородную связь с $12 \mathrm{NQ} ; \angle(=\mathrm{O} 1 \ldots \mathrm{H}-\mathrm{O})-$ валентный угол (deg); $\Delta \mathrm{H}(=\mathrm{O} 1 \ldots \mathrm{H}-\mathrm{O})$ - энтальпия образования водородной связи $(\mathrm{kcal} / \mathrm{mol})$ между атомом кислорода О1 молекулы $12 \mathrm{NQ}$ и атомом водорода молекулы воды.

а Разность энтальпий образования Н-комплекса $12 \mathrm{NQ}$ с двумя молекулами воды $6.03 \mathrm{kcal} / \mathrm{mol}$ и димера воды $2.54 \mathrm{kcal} / \mathrm{mol}$.

b Разность энтальпий образования Н-комплекса $12 \mathrm{NQ}$ с тремя молекулами воды $10.37 \mathrm{kcal} / \mathrm{mol}$ и тримера воды $5.46 \mathrm{kcal} / \mathrm{mol}$.

c Разность энтальпий образования Н-комплекса $12 \mathrm{NQ}$ с четырьмя молекулами воды $15.37 \mathrm{kcal} / \mathrm{mol}$ и тетрамера воды $11.10 \mathrm{kcal} / \mathrm{mol}$.

На основе данных этого же расчета получено, что наилучшее воспроизведение экспериментальных сдвигов полос поглощения молекулы $12 \mathrm{NQ}$ в растворе воды по сравнению с раствором в $H$-гексане наблюдается при расчете Н-комплексов $12 \mathrm{NQ}$ с димером и циклическим тетрамером воды, которые посредством водородных связей образуют замкнутую структуру с атомами кислорода карбонильных групп 12NQ.

Согласно данным расчета PCM TD-DFT B3LYP/6-311+G(d,p), около 70\% вклада в сдвиги первых трех полос поглощения молекулы 12NQ в воде по сравнению с раствором в $H$-гексане вносит континуум воды (общий эффект растворителя). Это позволяет сделать предположение о сопоставимом (или даже преобладающем) вкладе общего эффекта растворителя по сравнению со специфическим (образование водородных связей) в сдвиги этих полос поглощения $12 \mathrm{NQ}$ в водном растворе.

\section{Благодарности}

Работа выполнена на оборудовании Центра коллективного пользования „Спектр“ ИФМК УФИЦ РАН. Авторы также благодарят Центр коллективного пользования „Химия“ УфИХ УФИЦ РАН за предоставленную возможность использования суперкомпьютера для проведения квантово-химических расчетов.

\section{Финансирование работы}

Работа выполнена в рамках темы госзадания № AAAA-A19-119022290052-9.

\section{Конфликт интересов}

Авторы заявляют, что у них нет конфликта интересов.

\section{Список литературы}

[1] Reichardt C., Welton T. Solvents and Solvent Effects in Organic Chemistry. Weinheim: WILEY-VCH, 2011. 718 p.

[2] Dopfer O., Fujii M. // Chem. Rev. 2016. V. 116. N 9. P. 5432. doi 10.1021/acs.chemrev.5b00610

[3] Litwinienko G., Ingold K. // Acc. Chem. Res. 2007. V. 40. N 3. P. 222. doi $10.1021 / \operatorname{ar} 0682029$

[4] Cramer C.J., Truhlar D.G. // Chem. Rev. 1999. V. 99. N 8. P. 2161. doi $10.1021 / \mathrm{cr} 960149 \mathrm{~m}$

[5] Tomasi J., Persico M. // Chem. Rev. 1994. V. 94. N 7. P. 2027. doi 10.1021/cr00031a013

[6] Tomasi J., Mennucci B., Cammi R. // Chem. Rev. 2005. V. 105. N 8. P. 2999. doi 10.1021/cr9904009

[7] Miertus S., Scrocco E., Tomasi J. // Chem. Phys. 1981. V. 55. N 1. P. 117. doi 10.1016/0301-0104(81)85090-2

[8] Vetta M., Menger M.F.S.J., Nogueira J.J., Gonzalez L. // J. Phys. Chem. B. 2018. V. 122. N 11. P. 2975. doi 10.1021/acs.jpcb.7b12560

[9] Gustavsson T., Banyasz A., Lazzarotto E., Markovitsi D., Scalmani G., Frisch M.J., Barone V., Improta R. // J. Am. Chem. Soc. 2006. V. 128. N 2. P. 607. doi 10.1021/ja056181s

[10] Scalmani G., Frisch M.J., Mennucci B., Tomasi J., Cammi R., Barone V. // J. Chem. Phys. 2006. V. 124. P. 094107. doi 10.1063/1.217325

[11] Sancho M.I., Almandoz M.C., Blanco S.E., Castro E.A. // Int. J. Mol. Sci. 2011. V. 12. P. 8895. doi 10.3390/ijms 12128895

[12] Цеплин Е.Е., Цеплина С.Н., Хвостенко О.Г. // Опт. и спектр. 2018. Т. 125. № 4. С. 485. doi 10.21883/OS.2018.10.46699.104-18; Tseplin E.E., Tseplina S.N., Khvostenko O.G. // Opt. Spectrosc. 2018. V. 125. N 4. P. 506. doi $10.1134 / \mathrm{S} 0030400 \mathrm{X} 18100260$

[13] Tseplin E.E., Tseplina S.N. // Chem. Phys. Lett. 2019. V. 716. P. 142. doi 10.1016/j.cplett.2018.12.038

[14] Improta R., Barone V. // J. Am. Chem. Soc. 2004. V. 126. N 44. P. 14320. doi 10.1021/ja0460561

[15] Цеплин Е.Е., Цеплина С.Н., Хвостенко О.Г. // Опт. и спектр. 2011. T. 110. № 6. C. 956; Tseplin E.E., Tseplina S.N., Khvostenko O.G. // Opt. Spectrosc. 2011. V. 110. N 6. P. 903. doi 10.1134/S0030400X11060166 
[16] Цеплин Е.Е., Цеплина С.Н., Хвостенко О.Г. // Опт. и спектр. 2012. T. 113. № 4. C. 454; Tseplin E.E., Tseplina S.N., Khvostenko O.G. // Opt. Spectrosc. 2012. V. 113. N 4. P. 411. doi 10.1134/S0030400X12080206

[17] Цеплин Е.Е., Цеплина С.Н., Хвостенко О.Г. // Опт. и спектр. 2016. T. 120. № 2. C. 286; Tseplin E.E., Tseplina S.N., Khvostenko O.G. // Opt. Spectrosc. 2016. V. 120. N 2. P. 274. doi 10.1134/S0030400X16010203

[18] Martínez-Fernández L., Pepino A.J., Segarra-Martí J., Banyasz A., Garavelli M., Improta R. // J. Chem. Theory Comput. 2016. V. 12. P. 4430. doi 10.1021/acs.jctc.6b00518

[19] Pullman B., Miertius S., Perahia D. // Theor. Chim. Acta. 1979. V. 50. N 4. P. 317. doi 10.1007/BF00551339

[20] Bilkan M.T. // Phys. Chem. Liq. 2019. V. 57. N 1. P. 100. doi 10.1080/00319104.2018.1423564

[21] Gaigeot M.-P., Sprik M. // J. Phys. Chem. B. 2004. V. 108. N 22. P. 7458. doi 10.1021/jp049940m

[22] Van Mourik T. // Phys. Chem. Chem. Phys. 2001. V. 3. N 14. P. 2886. doi $10.1039 / \mathrm{B} 102701 \mathrm{H}$

[23] Fogarasi G., Szalay P.G. // Phys. Chem. Chem. Phys. 2015. V. 17. N 44. P. 29880 . doi $10.1039 /$ C5CP04563K

[24] Van Mourik T., Danilov V.I., Gonzalez E., Deriabina A., Poltev V.I. // Chem. Phys. Lett. 2007. V. 445. N 4-6. P. 303. doi 10.1016/j.cplett.2007.07.081

[25] Kim S., Schaefer H.F. // J. Chem. Phys. 2007. V. 126. N 6. P. 064301. doi 10.1063/1.2432123

[26] Nagakura S., Kuboyama A. // J. Am. Chem. Soc. 1954. V. 76. N 4. P. 1003. doi 10.1021/ja01633a017

[27] Kuboyama A., Yamazaki R., Yabe S., Uehara Y. // Bull. Chem. Soc. Jpn. 1969. V. 42. N 1. P. 10. doi 10.1246/bcsj.42.10

[28] Kuboyama A., Arano H. // Bull. Chem. Soc. Jpn. 1976. V. 49. N 5. P. 1401. doi 10.1246/bcsj.49.1401

[29] Kuboyama A., Matsumoto H. // Bull. Chem. Soc. Jpn. 1979. V. 52. N 6. P. 1796. doi 10.1246/bcsj.52.1796

[30] Kuboyama A. // Bull. Chem. Soc. Jpn. 1981. V. 54. N 3. P. 873. doi $10.1246 /$ bcsj. 54.873

[31] Becke A.D. // J. Chem. Phys. 1993. V. 98. N 7. P. 5648. doi 10.1063/1.464913

[32] Lee C., Yang W., Parr R.G. // Phys. Rev. B. 1988. V. 37. N 2. P. 785. doi 10.1103/PhysRevB.37.785

[33] Stephens P.J., Devlin F.J., Chabalowski C.F., Frisch M.J. // J. Phys. Chem. 1994. V. 98. N 45. P. 11623. doi $10.1021 / \mathrm{j} 100096 \mathrm{a} 001$

[34] Frisch M.J., Trucks G.W., Schlegel H.B., Scuseria G.E., Robb M.A., Cheeseman J.R., Scalmani G., Barone V., Mennucci B., Petersson G.A., Nakatsuji H., Caricato M., Li X., Hratchian H.P., Izmaylov A.F., Bloino J., Zheng G., Sonnenberg J.L., Hada M., Ehara M., Toyota K., Fukuda R., Hasegawa J., Ishida M., Nakajima T., Honda Y., Kitao O., Nakai H., Vreven T., Montgomery J.A. Jr, Peralta J.E., Ogliaro F., Bearpark M., Heyd J.J., Brothers E., Kudin K.N., Staroverov V.N., Kobayashi R., Normand J., Raghavachari K., Rendell A., Burant J.C., Iyengar S.S., Tomasi J., Cossi M., Rega N., Millam J.M., Klene M., Knox J.E., Cross J.B., Bakken V., Adamo C., Jaramillo J., Gomperts R., Stratmann R.E., Yazyev O., Austin A.J., Cammi R., Pomelli C., Ochterski J.W., Martin R.L., Morokuma K., Zakrzewski V.G., Voth G.A., Salvador P., Dannenberg J.J., Dapprich S., Daniels A.D., Farkas O., Foresman J.B., Ortiz J.V., Cioslowski J., Fox D.J., Gaussian 09, Revision C.1, Gaussian, Inc., Wallingford CT, 2009.
[35] Zhurko G.A., Zhurko D.A. Chemcraft version 1.7 [Электронный ресурс] Режим доступа: https://www.chemcraftprog.com 